\title{
Focus on Orientation to Represent Terrain
}

\author{
Gene Trantham ${ }^{a}$, Patrick Kennelly ${ }^{b}$ \\ ${ }^{a}$ Pennsylvania State University, gzt5142@psu.edu \\ ${ }^{b}$ Long Island University, Patrick.Kennelly@liu.edu \\ * Corresponding author
}

Keywords: Relief Shading; Bump-Mapping; Terrain Representation; Surface Normal Vectors

\begin{abstract}
:
The most often used and powerful terrain representation layers symbolize the orientation of the surface rather than its elevation. Techniques such as relief shading and slope-shading symbolize metrics of orientation, yet typically ingest elevation data to create those layers. Rather than processing elevation data to represent terrain, a shift in perspective to emphasize orientation data - surface normal vectors - can dramatically speed some analyses and opens the door to new techniques borrowed from the computer graphics field. Performance improvements are most apparent for multidirectional methods, making those techniques more available to cartographers with modest computing resources.
\end{abstract}

Elevation is the dominant data model for terrain surfaces, and the de-facto input for widely available analytic cartography tools. It is the essential property driving hypsometric tinting or viewshed analyses. Yet the most widely used layers for terrain representation do not symbolize elevation, but rather orientation. These analyses depend on an interpretation of orientation from the elevation data. Each tool must do this on its own and may choose its own method for doing so. Complex techniques built upon relief shading or slope shading, such as Sky Model (Kennelly \& Stewart, 2011) found in Esri's TerrainTools, perform this interpretation many times. This is duplicated work, and largely unnecessary. If adjacent tools interpret orientation differently, incongruencies introduce bias or noise to the analysis.

An orientation-focused data model is available, widely used in other industries but under-used in GIS. This is a multiband raster dataset encoding surface normal vectors. Surface normal vectors completely capture pixel-by-pixel orientation independent of height and can be used directly in more modular and streamlined versions of the tools now common. This model separates elevation-to-orientation processing and executes only once, forming a persistent dataset to be fed to further analyses. The orientation calculations, because they are separate, can also be customized to the terrain type. When a tool such as Hillshade is unencumbered from this additional processing, it becomes more lightweight and executes faster, with less overhead.

Multi-directional shading methodologies will benefit from improved performance in proportion to the number of lighting directions in the method. Sky Model runs the shading tool hundreds of times to accumulate a composite image (shown in Figure 1, compared with a traditional single-light shaded relief). An orientation-aware version runs several times faster than the widely available versions built atop conventional shading tools.
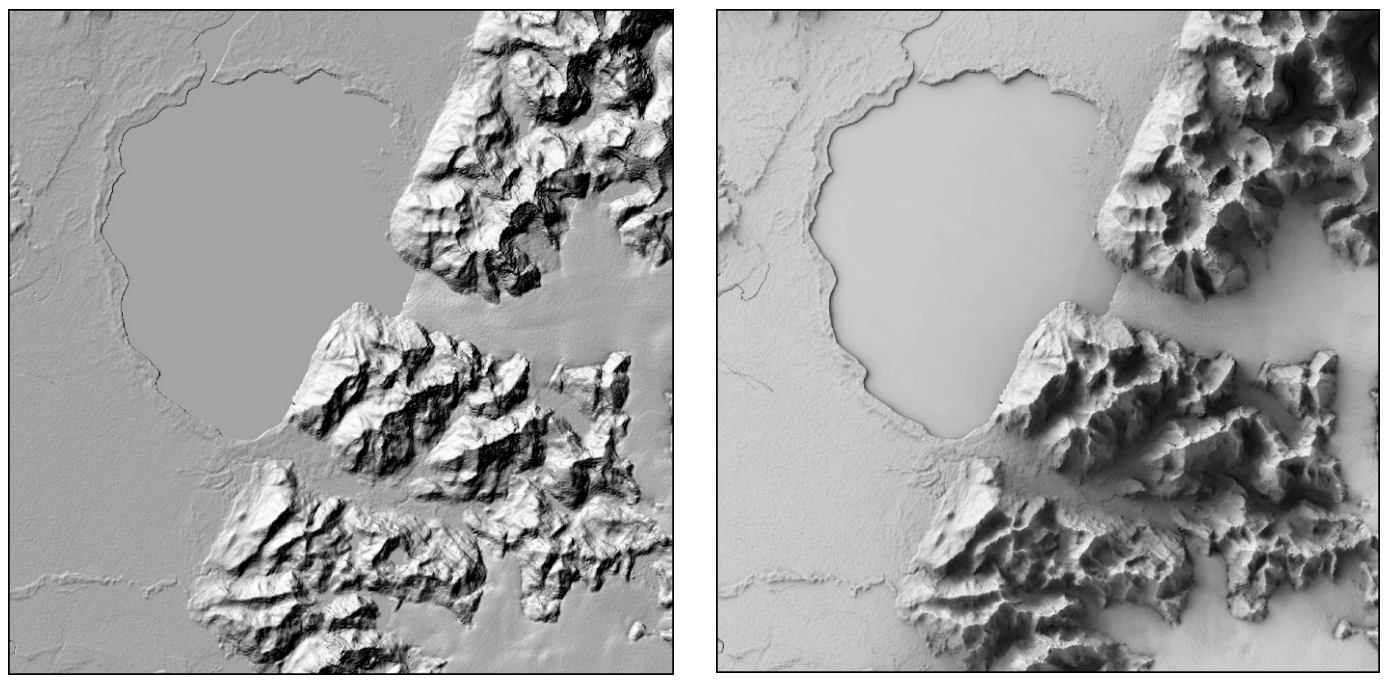

Figure 1: Conventional Hillshade vs. Sky Model for Laguna San Rafael, Chile 
Moving to a surface normal vector dataset permits us to implement proven techniques from the computer graphics field for novel cartographic effects. A version of displacement mapping has been used in GIS to raise patterns on a surface (Nighbert, 2002). This is a manipulation of the DEM. Bump-mapping produces a similar effect by operating on the surface normal vectors rather than the height data (Blinn, 1978). Bump-mapping, when employed with effective masking, is a useful technique to imprint textures onto the terrain that are themselves shaded properly. Critically, surface normal vector manipulations do not affect elevation analyses (hypsometric tints, shadows, etc). Patterns introduced to the relief shading, working alone or with other variables such as colour, are a means of differentiating distinct areas in the scene. Figure 2 illustrates the bump-mapping effect to create a textured shaded relief in which land use categories (forest and open water in this case) can be identified by colour, texture, or both.

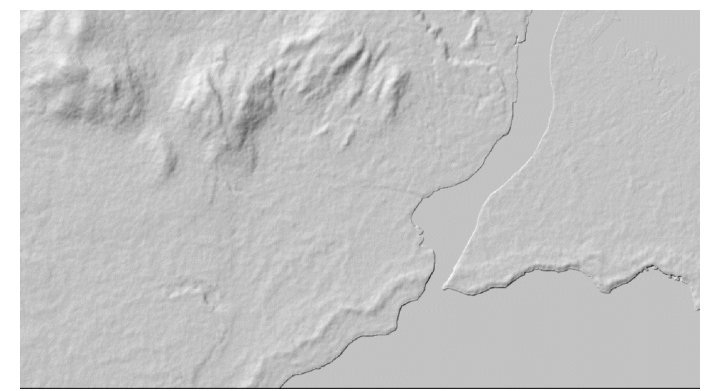

(a) Traditional shaded relief, without bump-mapping

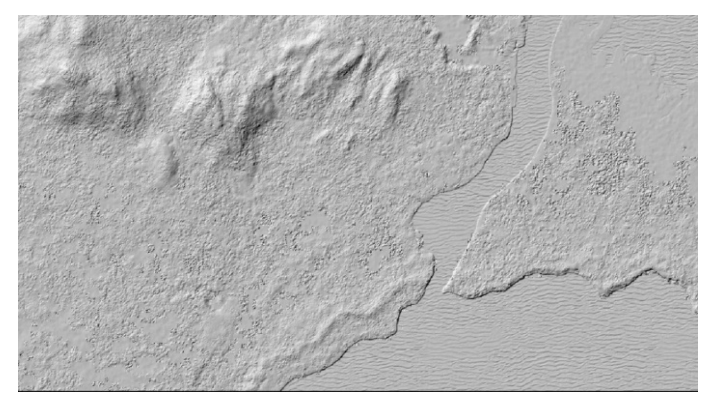

(c) Bump-mapped textures applied

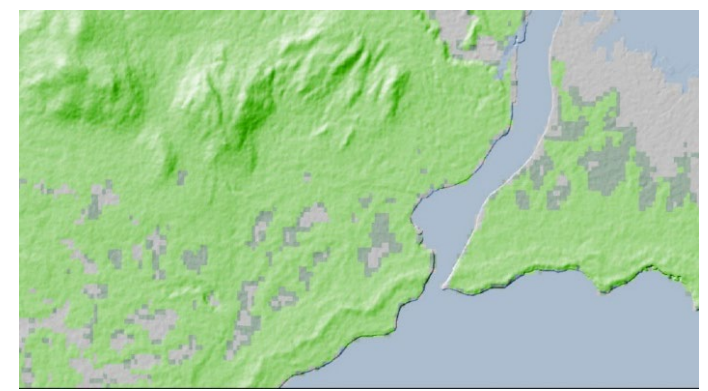

(b) Land cover data defining forested and water areas

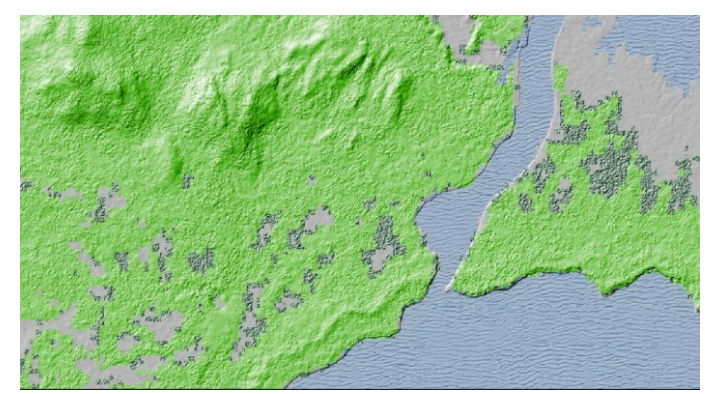

(c) Bump-mapped textures, with land cover data supplying colour

Figure 2. Conventional vs. Bump-Mapped Shaded Relief for Laguna San Rafael, Chile 